\title{
Rede social de pessoas que fazem uso abusivo de álcool ao sul do Brasil
}

\author{
Social network of people with alcohol abuse disorders in southern Brazil \\ Red social de personas que hacen abuso de alcohol en el sur de Brasil
}

Recebido: 1511/2021 | Revisado: 22/11/2021 | Aceito: 27/11/2021 | Publicado: 09/12/2021

Martha Lettnin Haertel Moreira
ORCID: https://orcid.org/0000-0001-8869-2694
Universidade Federal de Pelotas, Brasil
E-mail: marthahaertel@ hotmail.com
Vanda Maria da Rosa Jardim
ORCID: https://orcid.org/0000-0001-8320-4321
Universidade Federal de Pelotas, Brasil
E-mail: vandamrjardim@gmail.com
Valeria Cristina Christello Coimbra
ORCID: https://orcid.org/0000-0001-5327-0141
Universidade Federal de Pelotas, Brasil
E-mail:valeriacoimbra@ @otmail.com
Elitiele Ortiz dos Santos
ORCID: https://orid.org/0000-0002-2537-6069
Universidade Federal do Pampa, Brasil
E-mail:elitielesantos@ unipampa.edu.br
Liamara Denise Ubessi
ORCID: https://orcid.org/0000-0002-5884-9969
Universidade Federal de Pelotas, Brasil
E-mail:liaubessi @gmail.com

\section{Resumo}

Objetivo: analisar a rede social de pessoas que fazem uso abusivo de álcool assistidas na Rede de Atenção Psicossocial. Método: pesquisa qualitativa, realizada com pessoas em uso problemático de álcool em um município de pequeno porte do Rio Grande do Sul, Brasil. A coleta de dados ocorreu em 2014 através de entrevistas semiestruturadas, ecomapa e análise de documentos. Para análise dos dados, foi utilizada a análise temática. Resultados: a rede social inclui família, amigos, Centro de Atenção Psicossocial álcool e outras drogas (CAPSad), espaço de geração de renda, trabalho, escola, ambiente domiciliar e atividades de lazer. O CAPSad é um importante recurso na rede social, pelo apoio no tratamento e por suprir necessidades como alimentação, medicação, socialização e afeto. Conclusão: identifica-se uma rede social diversificada, com recursos comunitários que auxiliam na manutenção da espiritualidade, na inserção no mercado de trabalho através da profissionalização e nos serviços de saúde, que contribuem para a manutenção do tratamento.

Palavras-chave: Transtornos relacionados ao uso de álcool; Apoio social; Saúde mental.

\begin{abstract}
Objective: to analyze the social network of people with alcohol abuse disorders assisted in the Psychosocial Care Network. Methods: qualitative research, carried out with people in problematic use of alcohol in a small city of Rio Grande do Sul, Brazil. Data collection took place in 2014 through semi-structured interviews, ecomap, and document analysis. For data analysis, thematic analysis was used. Results: the social network includes family, friends, the Alcohol and Other Drug Psychosocial Care Center (CAPSad), the income generation space, work, school, home environment and leisure activities. The CAPSad is an important resource in the social network for supporting treatment, in addition to meeting needs such as food, medication, socialization and affection. Conclusion: a diversified social network is identified, with community resources that help maintain spirituality, enter the labor market through professional training, and health services, which contribute to treatment maintenance.
\end{abstract}

Keywords: Alcohol-related disorders; Social support; Mental health.

\section{Resumen}

Objetivo: analizar una red social de personas que abusan del alcohol atendidas en la Red de Atención Psicosocial. Método: investigación cualitativa, realizada con personas con consumo problemático de alcohol, en una pequeña ciudad de Rio Grande do Sul, Brasil. La recolección de datos se realizó en 2014 a través de entrevistas semiestructuradas, ecomap y análisis de documentos. Para el análisis de datos se utilizó el análisis temático. Resultados: la red social incluye familia, amigos, Centro de Atención Psicosocial alcohol y otras drogas (CAPSad), espacio para la generación de ingresos, trabajo, escuela, ambiente del hogar y actividades de ocio. El CAPSad es un recurso importante en la red social para apoyar el tratamiento y satisfacer necesidades como alimentación, 
medicación, socialización y afecto. Conclusión: se identifica una red social diversificada, con recursos comunitarios que ayudan a mantener la espiritualidad, ingresar al mercado laboral a través de la formación profesional y servicios de salud, que contribuyen al mantenimiento del tratamiento.

Palabras clave: Trastornos relacionados con alcohol; Apoyo social; Salud mental.

\section{Introdução}

O consumo prejudicial de substâncias psicoativas, álcool ou outras drogas surge no contexto contemporâneo como um problema de saúde pública, com a necessidade de compreensão e práticas baseadas na complexidade desse fenômeno.

No uso abusivo de álcool, as consequências são diversas, relacionadas a mais de 60 tipos de doenças, além de episódios de violência, homicídios e acidentes. Estima-se que uma a cada 20 mortes é relacionada ao uso prejudicial de álcool, totalizando 3,3 milhões de mortes no mundo (World Health Organization, 2018). No Brasil, no período de 2012 a 2016 , foram registrados 33.168 óbitos atribuíveis ao uso de álcool. Essa elevada mortalidade preocupa, pois o uso do álcool deve ser considerado como causa evitável para mortes prematuras (Marques et al., 2020).

Diante disso, diversos países vêm procurando respostas efetivas às crescentes repercussões sociais, cognitivas, culturais e políticas do consumo prejudicado de álcool. Desde 2003, pela Política Nacional de Atenção Integral a Usuários de Álcool e Outras Drogas, tornou-se imperativa a necessidade de estruturação de uma rede centrada na comunidade com ênfase na reabilitação e reinserção social, além de redução de danos com estratégia principal de trabalho (Silva \& Abrahão, 2019).

A dimensão complexa do problema evidencia a necessidade do trabalho em rede e intervenções que não foquem na substância, mas que ampliem o olhar para a pessoa e o conjunto de relações que a cerca no território, como família e serviços, na sua rede social.

A rede social se constitui das relações do indivíduo com aquilo que interage no seu cotidiano, no qual pode ser observado os recursos acionados, a forma de relação, os fluxos e como a pessoa é afetada por eles. A função da rede se relaciona à qualidade das relações, sendo que o apoio social inclui o apoio emocional, financeiro, material, de informação e a interação social positiva. O apoio social pode impactar positivamente na diminuição do uso de substâncias psicoativas (Souza et al., 2011, Tan et al., 2021, Fitzpatrick, 2016).

Em relação à problemática do uso de drogas, compreende-se que a identificação dos componentes de apoio dentro da rede social permite problematizar questões mais complexas que aquelas limitadas a doença. Além disso, o processo de dependência culmina em estresses relacionais nas mais diversas esferas, como o trabalho, família, amigos e vizinhos, exigindo intervenções que contemplem esses contextos (Souza, et al., 2011).

Assim, analisar a rede social das pessoas que fazem uso abusivo de álcool vai possibilitar maior compreensão dessa experiência e identificação de elementos de apoio e conflito, que podem facilitar e qualificar as ações de cuidado e prevenção. Nesse sentido, o estudo tem por objetivo analisar a rede social de pessoas que fazem uso abusivo de álcool assistidos na Rede de Atenção Psicossocial de um município do Rio Grande do Sul.

\section{Metodologia}

Estudo descritivo e analítico de abordagem qualitativa. A pesquisa qualitativa busca compreender as diversas interpretações dos sujeitos frente aos aspectos da vida, à complexidade e singularidades das relações, valores, crenças, percepções, cultura, enfim, um universo de significados que não pode ser quantificado (Minayo, 2010).

O estudo foi desenvolvido com sete pessoas em uso problemático de álcool inseridas na Rede de Atenção Psicossocial (RAPS) do município de pequeno porte do estado do Rio Grande do Sul, com população estimada de 44.561 habitantes (IBGE, 2017). O nome do município não será informado, para fins de preservação da identidade dos participantes. 
A coleta de dados foi realizada no período de julho a dezembro de 2014. Utilizaram-se como instrumentos de coleta de dados as entrevistas semiestruturadas e o ecomapa, com foco nos aspectos relacionados ao cotidiano do usuário, locais que frequenta, relações e a rede de suporte. Além disso, foi utilizada a análise documental de prontuários arquivados no Centro de Atenção Psicossocial Álcool e outras Drogas, para conhecer a trajetória da pessoa durante o tratamento.

Para a seleção dos participantes, foi realizada entrevista com um informante chave, nesse caso, uma pessoa com problemas relacionados ao uso de álcool e que percorreu diferentes trajetórias na RAPS. Para seleção dos demais entrevistados, utilizou-se a técnica de bola de neve, ou seja, cada participante indicou outro considerado apto a contribuir com os objetivos da pesquisa, e assim sucessivamente.

O ecomapa é uma das ferramentas qualitativas preconizadas pelo Guia para Avaliação e Intervenção na Família, com o objetivo de obter informações sobre a rede social. Assim, trouxe contribuições importantes para o entendimento sobre quem faz parte dessa rede e os vínculos desenvolvidos (Wright \& Leahey, 2012).

Os critérios de seleção dos participantes foram ser dependente químico de álcool, receber indicação de outro entrevistado. Os participantes foram identificados com nomes fictícios, seguido do número da entrevista, por exemplo, Sujeito1 (S1) - Mirelle, e assim sucessivamente, com respeito ao anonimato.

A análise dos dados foi realizada pela análise temática em três etapas. Na primeira etapa, pré-análise, foi realizada a leitura exaustiva do material coletado. Na segunda etapa, ocorreu a exploração do material, com a separação dos fragmentos, os quais foram distribuídos em tópicos, identificados como unidades de informação; em seguida, foram aproximadas dando origem à seguinte categoria analítica: Rede social das pessoas que fazem uso abusivo de álcool. E, por fim, na terceira etapa, foi realizada uma síntese interpretativa dos resultados obtidos (Minayo, 2010).

A pesquisa foi aprovada pelo Comitê de Ética da Faculdade de Enfermagem da UFPel sob o Parecer n ${ }^{\circ} 705.163 / 2014$, respeitando os princípios éticos conforme a Resolução 466/2012 do Conselho Nacional de Saúde.

\section{Resultados}

\section{Rede social das pessoas que fazem uso abusivo de álcool}

As pessoas em uso abusivo de álcool desta pesquisa eram quatro homens e três mulheres, com a faixa etária de 36 a 71 anos. Quatro se autodeclaram brancos, e três, negros. Quanto à escolaridade, três apresentam ensino fundamental incompleto, dois, ensino médio incompleto, um deles, ensino médio completo, e outro, curso superior completo. Quatro moram com os filhos e/ou companheiro (a) e três moram sozinhos. Referente à ocupação, três possuem trabalho formal e dois recebem auxílio doença, dois são aposentados. Seis participantes recebem um salário mínimo e um recebe meio salário mínimo.

Entre as perdas relatadas pelo uso abusivo do álcool, três relataram perda de emprego ou de oportunidade de trabalho. Dois relataram a perda de uma relação harmoniosa com os filhos e uma relatou a perda da guarda da filha. Entre os tratamentos anteriores, analisaram-se nos registros dos prontuários internações em Comunidades Terapêuticas, em enfermarias especializadas para tratamento de drogas em hospitais gerais, CAPSad e também tratamentos em outros municípios. Com relação ao tratamento atual, cinco fazem acompanhamento no CAPSad, um faz acompanhamento apenas na Atenção Básica e um deles está inserido no espaço de Oficinas de Geração de Renda, denominado 'Lokomotiva'.

Na Figura 1, pode ser observado o ecomapa de um dos sujeitos da pesquisa, fornecendo uma visão das relações com as instituições e outras pessoas, e a natureza dessas relações (vínculo mais forte, forte, fraco, e vínculo conflituoso). Com o ecomapa, foi possível avaliar os vínculos com a rede social e explorar o suporte na elaboração de estratégias de cuidado. 
Figura 1 - Ecomapa das relações do Sujeito 1, Rio Grande do Sul, Brasil, 2014.

Legenda

Intensidade da relação

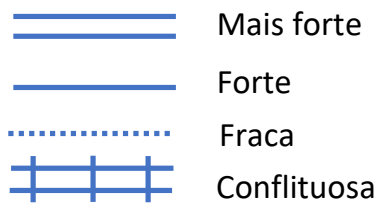

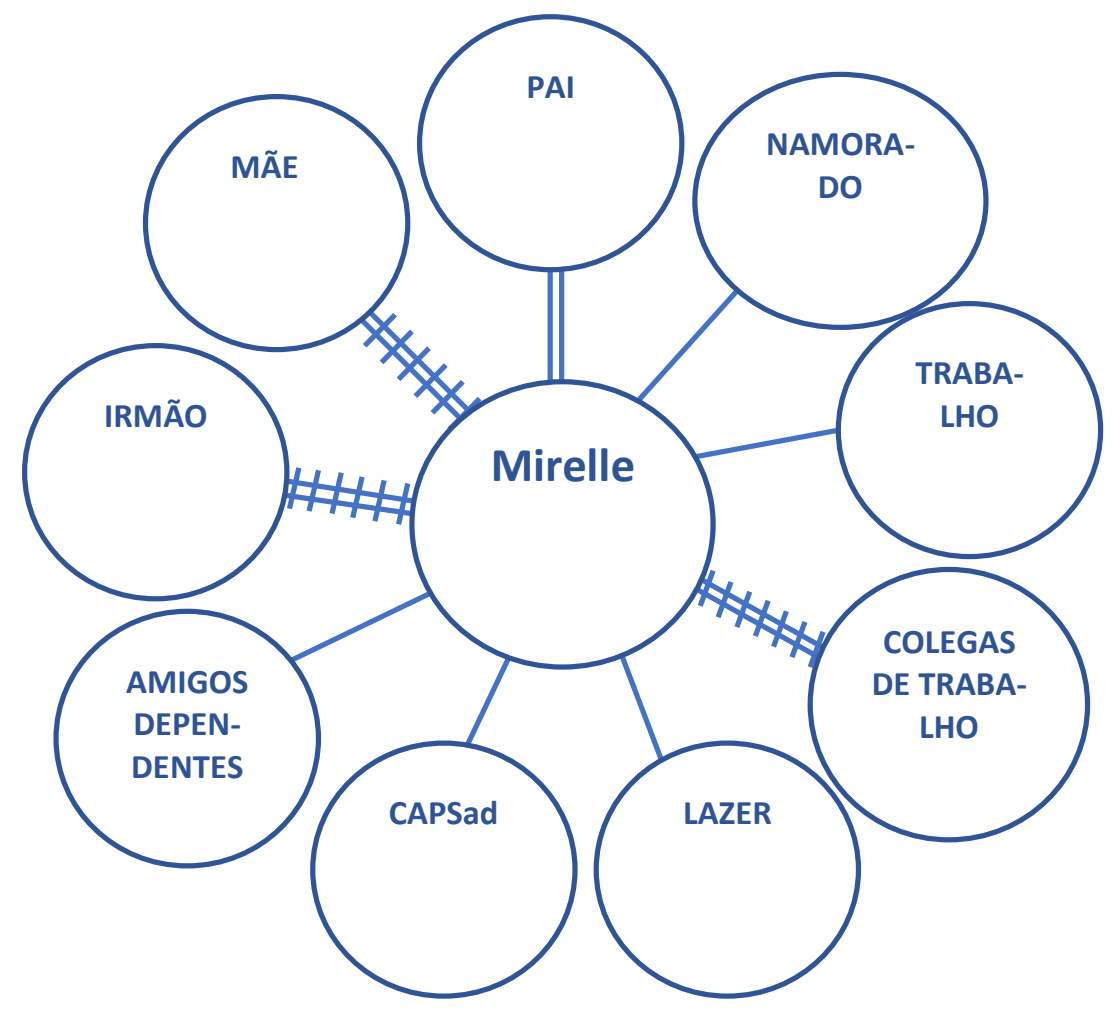

Fonte: Autores (2021).

No Quadro 1, pode ser observada a síntese das interações sociais dos participantes com a identificação dos componentes rede, os vínculos e o tipo de apoio.

Quadro 1 - Síntese das interações sociais de sujeitos que fazem uso abusivo de álcool. Rio Grande do Sul, Brasil, 2014.

\begin{tabular}{|c|c|c|c|c|}
\hline $\begin{array}{l}\text { Rede } \\
\text { social }\end{array}$ & Vínculos mais fortes & Vínculos fortes & Vínculo fraco & Vínculo conflituoso \\
\hline $\begin{array}{l}\text { S1 - } \\
\text { Mirelle }\end{array}$ & Pai - compreensão, apoio & $\begin{array}{l}\text { Amigos dependes químicos - } \\
\text { compreensão, ajuda mútua } \\
\text { CAPSad - local que se sente bem, } \\
\text { rever questões pessoais e } \\
\text { profissionais, ajudar os outros } \\
\text { usuários } \\
\text { Lazer - pescar, visitar os pais, } \\
\text { cuidar do jardim } \\
\text { Trabalho - realização profissional, } \\
\text { dedicação, novos aprendizados } \\
\text { Namorado - companheirismo, } \\
\text { apoio material, emocional e nas } \\
\text { tarefas diárias }\end{array}$ & $\begin{array}{ll}\text { Vínculo } & \text { não } \\
\text { identificado } & \end{array}$ & $\begin{array}{l}\text { Irmão - acusação } \\
\begin{array}{l}\text { Mãe - acusação, culpa pelo uso } \\
\text { do irmão }\end{array} \\
\begin{array}{l}\text { Colegas de trabalho - } \\
\text { preconceito }\end{array}\end{array}$ \\
\hline $\begin{array}{l}\text { S2 } \\
\text { Ana }\end{array}$ & $\begin{array}{l}\text { Casa - proteção quando sente } \\
\text { vontade de beber } \\
\text { Filho - apoio afetivo, financeiro e } \\
\text { nas tarefas domésticas } \\
\text { Filha - apoio afetivo } \\
\text { CAPSad - apoio nos momentos } \\
\text { difíceis, bons relacionamentos }\end{array}$ & $\begin{array}{l}\text { Igreja - encontra forças para } \\
\text { manter abstinência } \\
\text { Lazer } \\
\text { Vizinhos } \\
\text { Namorado }\end{array}$ & $\begin{array}{l}\text { Amigos usuários de } \\
\text { drogas - risco para } \\
\text { recair }\end{array}$ & $\begin{array}{l}\text { Irmã - incentiva e oferece } \\
\text { bebida }\end{array}$ \\
\hline
\end{tabular}




\begin{tabular}{|c|c|c|c|c|}
\hline $\begin{array}{l}\text { S3 - } \\
\text { Celina }\end{array}$ & $\begin{array}{l}\text { Escola - motivação para alcançar os } \\
\text { objetivos futuros } \\
\text { Amigos usuários de drogas- } \\
\text { descontração; se sente aceita, } \\
\text { valorizada, e fazem uso de bebida }\end{array}$ & $\begin{array}{l}\text { Lazer } \\
\text { CAPSad - se sente aceita, } \\
\text { valorizada, produção } r \\
\text { artesanato que ajudam na renda } \\
\text { familiar }\end{array}$ & $\begin{array}{l}\text { Vínculo } \\
\text { identificado }\end{array}$ & $\begin{array}{l}\text { Filhos/marido - não aceitam o } \\
\text { uso, há acusação e agressão } \\
\text { verbal, não apoiam o } \\
\text { acompanhamento no CAPSad } \\
\text { Trabalho - autonomia } \\
\text { financeira, exploração, sem a } \\
\text { garantia de direitos trabalhistas }\end{array}$ \\
\hline $\begin{array}{l}\text { S4 - } \\
\text { Carlos }\end{array}$ & $\begin{array}{l}\text { Casa - estratégia de proteção devido } \\
\text { à fobia social, satisfação em } \\
\text { trabalhar na sua marcenaria } \\
\text { Esposa - companhia } \\
\text { Irmãos - apoio, acompanhamento } \\
\text { no tratamento }\end{array}$ & $\begin{array}{l}\text { Lazer - passear de carro na praia, } \\
\text { ir no mercado, compras, assistir } \\
\text { televisão }\end{array}$ & $\begin{array}{l}\text { CAPSad - críticas à } \\
\text { forma de tratamento- } \\
\text { equipe precisa ser } \\
\text { mais rigorosa com os } \\
\text { usuários, inseri-los } \\
\text { nas atividades } \\
\text { domésticas, não } \\
\text { aceitar que pessoas } \\
\text { em tratamento } \\
\text { continuem usando } \\
\text { drogas } \\
\begin{array}{l}\text { Amigos - não gosta } \\
\text { de estar com os } \\
\text { amigos, fobia social }\end{array}\end{array}$ & Vínculo não identificado \\
\hline $\begin{array}{l}\text { S5 - } \\
\text { Vagner }\end{array}$ & $\begin{array}{l}\text { Esposa - apoio, afeto } \\
\text { Filha - apoio, companhia } \\
\text { Cunhado/sogra - apoio } \\
\text { Igreja - superação da perda do filho, } \\
\text { satisfação em integrar coral da } \\
\text { igreja } \\
\text { Lokomotiva - profissionalização, } \\
\text { amizades } \\
\text { CAPSad - círculo de amizades }\end{array}$ & $\begin{array}{l}\text { Unidade Básica de Saúde } \\
\text { tratamento para hipertensão e } \\
\text { renovação de receitas } \\
\text { Trabalho - satisfação na padaria, } \\
\text { artesanato e como eletricista }\end{array}$ & $\begin{array}{ll}\text { Vínculo } & \text { não } \\
\text { identificado } & \end{array}$ & $\begin{array}{l}\text { Irmãos - conflitos devido à } \\
\text { divisão de herança }\end{array}$ \\
\hline $\begin{array}{l}\text { S6 - } \\
\text { Jeremia } \\
\text { s }\end{array}$ & $\begin{array}{l}\text { Irmãos adotivos - apoio } \\
\text { Sobrinha - apoio } \\
\text { Amigos - companhia, conversa } \\
\text { Lokomotiva - profissionalização, } \\
\text { círculo de amizades }\end{array}$ & $\begin{array}{l}\text { Lazer - passear nos amigos, } \\
\text { participar de eventos, vender seus } \\
\text { produtos }\end{array}$ & $\begin{array}{l}\text { CAPSad } \\
\text { alimentação, } \\
\text { medicação, } \\
\text { amizades. Teve alta } \\
\text { do serviço }\end{array}$ & Vínculo não identificado \\
\hline $\begin{array}{l}\text { S7 } \\
\text { Joaqui } \\
\text { m }\end{array}$ & $\begin{array}{l}\text { Caps AD - "segunda casa", fonte de } \\
\text { afeto, alimentação, amizades. } \\
\text { Participação nas oficinas, assiste } \\
\text { televisão, recebe apoio nas } \\
\text { atividades de matemática da escola } \\
\text { e ajuda no cuidado do serviço }\end{array}$ & $\begin{array}{l}\text { Escola - aprendizado, perspectiva } \\
\text { de futuro, círculo de amizades, se } \\
\text { sente acolhido } \\
\text { Trabalho }\end{array}$ & $\begin{array}{l}\text { Lazer - pescar com } \\
\text { os amigos, assistir } \\
\text { jogo de futebol } \\
\text { Amigos }\end{array}$ & $\begin{array}{l}\text { Filhos - era agressivo com os } \\
\text { filhos devido ao álcool }\end{array}$ \\
\hline
\end{tabular}

Fonte: Autores (2021).

\section{Discussão}

Neste estudo, a família aparece tanto como vínculo mais forte como conflituoso. A família, quando identificada como um dos vínculos mais fortes, é caracterizada como fonte de apoio afetivo, auxílio financeiro, auxílio nas tarefas domésticas e companhia.

Por outro lado, quando a família é considerada um vínculo conflituoso, são descritas dificuldades de relacionamento com situações de agressão verbal, agressão do usuário à família nos momentos que está em uso de álcool, falta de compreensão da família sobre o alcoolismo e o tratamento, e quando incentiva ao uso de álcool. A relação harmoniosa com a família é considerada uma das principais perdas relatadas pelo uso de substância psicoativa.

O uso prejudicado de drogas afeta a família. Identifica-se, inicialmente, distanciamento do usuário do convívio familiar, que, aos poucos, soma-se à agressividade, medo, dificuldade em dialogar, criando uma atmosfera de impotência, 
sobrecarga e insegurança (Rodrigues et al., 2018). Também há o estigma e o preconceito na própria família e sociedade, que causam sentimentos de culpa, vergonha e revolta, que fragilizam e podem adoecer a família.

Estudos apontam que a inclusão da família no processo de cuidado diminui o sofrimento familiar, promove melhorias nas relações e na qualidade de vida da família. Essa participação é fundamental para a recuperação do usuário, favorecendo a permanência e adesão ao tratamento, além da redução de internações prolongadas (Alvarez et al, 2012, Belotti; Fraga; Belotti, 2017).

Os serviços sociais e de saúde podem promover o cuidado à família e condições para a sua participação no cuidado (Ferreira et al, 2019). O comprometimento da família exige nova organização familiar e aquisição de habilidades que, talvez, desestruture as atividades diárias no primeiro momento. $\mathrm{O}$ apoio familiar proporciona segurança à pessoa em uso abusivo de drogas, bem como recurso para enfrentar as pressões dos grupos que forjam o consumo (Saiz et al, 2020).

A religião é outro vínculo de apoio da rede social que aparece neste estudo. É considerada um auxílio na superação dos momentos difíceis, na manutenção do controle do uso e possibilidade de interação com outras pessoas. As mensagens religiosas auxiliam a pessoa em uso abusivo do álcool refletir sobre os aspectos de sua vida, suas atitudes, encontrar soluções para enfrentar os problemas e alívio emocional (Beraldo et al, 2019, Zerbetto et al, 2017).

Portanto, compreender a dimensão da religião e espiritualidade na vida da pessoa e instrumentalizá-los a utilizar essa estratégia tem benefícios não só para manter o controle do uso, mas para auxiliar o usuário a manejar os problemas da vida e as relações (Beraldo et al, 2019, Zerbetto et al, 2017).

Para a reabilitação psicossocial, é importante considerar a contratualidade da pessoa no seu habitat, rede social e trabalho, assim inserindo-a socialmente (Kantorski et al, 2020). Neste estudo, os participantes destacam os vínculos com o trabalho, sendo considerada uma fonte de realização e satisfação profissional. Já o vínculo conflituoso com o trabalho é relacionado ao preconceito dos colegas e à falta de garantia dos direitos trabalhistas. Além disso, o trabalho é outro recurso social que aparece neste estudo como uma das perdas relatadas pelo uso de substância.

A perda dos vínculos com o trabalho é recorrente na vida dos usuários. Muitas vezes, agrava o problema da dependência devido à vulnerabilidade social, dificuldade de inserção em um novo trabalho, fragilização das relações familiares e sociais na reorganização financeira da família (Bard et al, 2016).

O trabalho é um meio que possibilita a reabilitação psicossocial ao ser humano, reproduzir sua vida material para satisfazer suas necessidades básicas, como comer, vestir, morar, e suprir as necessidades de sua vida social, o lazer, a convivência e a liberdade (Martins et al, 2018). Desse modo, o trabalho não pode estar desvinculado da vida do sujeito, da sua experiência.

A utilização do trabalho nas propostas de reabilitação psicossocial deve se configurar como um instrumento de inclusão social e promoção da cidadania (Sanches \& Vecchia, 2018). Isso implica construir estratégias, para facilitar o acesso ao mercado de trabalho, direitos trabalhistas e reorganização inicial para o autocuidado, visando uma preparação para inclusão nesse meio.

Além de se considerar a centralidade do trabalho na vida da pessoa, é importante resgatá-lo como operador de saúde mental. As abordagens tradicionais sobre tratamento e prevenção de álcool e drogas, em geral, restringem o problema à esfera doméstica. De certa forma, são intervenções sobre as doenças em detrimento do investimento em ações ético-políticas que considerem o trabalho o vetor para a construção da cidadania (Silva \& Ferigato, 2017).

Quanto à inserção no mundo do trabalho, neste estudo, a Lokomotiva é destaca como um dos vínculos mais fortes. A Lokomotiva é um Centro Integrado de Reabilitação Laboral de Saúde Mental vinculado ao Sistema Único de Saúde, que promove ações em saúde, trabalho, educação e inclusão, integrando as políticas de saúde mental, economia solidária e cultura. Oferece cursos de panificação, eletricista e artesanato em palha de milho. 
Para os participantes do estudo, a Lokomotiva possibilita a inserção no trabalho formal, ampliando os caminhos para a inclusão social, com a articulação de novas possibilidades, aprendizado, renda e sociabilidade.

As iniciativas, como a da Lokomotiva, ainda são incipientes no Brasil. Há, aproximadamente, 1.008 iniciativas no campo do trabalho e geração de renda, sendo que 57 delas estão localizadas no Rio Grande do Sul. Trata-se de um número aquém da necessidade, uma vez que o trabalho deve ser prioritário para as pessoas em desvantagem social (Ministério da Saúde, 2015).

Esse movimento esbarra em desafios que envolvem dificuldades de financiamento, atuação na informalidade, mas, mesmo assim, houve uma ampliação da economia solidária no país que se relaciona com a expansão da rede social das pessoas assistidas em saúde mental (Mazaro et al., 2020). Contudo, muitas vezes, evidencia-se falta de conhecimentos sobre empreendimentos solidários e autogestionários e falta de preparo dos profissionais para acolher pessoas que fazem uso abusivo de drogas, que confundem o cuidar com o ter poder sobre a vida dessas pessoas (Silva \& Ferigato, 2017, Mazaro et al., 2020).

Outro ponto da rede social dos participantes da pesquisa é o CAPSad, onde a pessoa se sente aceita e valorizada. O serviço também é aparece como vínculo importante que supre a necessidades de alimentação e higiene. A participação nas oficinas terapêuticas e atendimentos individuais são destacados como momentos para repensar questões pessoais, ajuda mútua e aprendizados. O vínculo fraco com o serviço ocorre devido à percepção de que a equipe deveria ser mais "rigorosa" e acolher somente usuários em abstinência.

As ações do CAPSad devem ser orientadas para a singularidade dos usuários, com uso de estratégias para redução de danos, sem imposição de tratamentos ou pré-requisitos de acesso ao serviço. Tal forma de trabalho já demonstra resultados, como a redução de agravos sociais e de saúde, e melhor adesão às ações (Fagundes Junior et al., 2016).

O CAPSad é um serviço estratégico no processo de reforma psiquiátrica, e aparece como uma das principais redes operantes entre as vinculações dos usuários de drogas (Silva et al.,). É um local onde os usuários se sentem acolhidos e reconhecidos como pessoas (Romanini et al., 2017). Nesse sentido, cabe ao CAPSad estabelecer as pontes necessárias entre o serviço, o território e as outras redes sociais, ampliando as possibilidades de intervenção sobre as necessidades sociais, de saúde e inserção social.

O CAPSad ainda precisa avançar na sua proposta de ser um local de passagem que presta atendimento à crise e casos graves, bem como de qualificar as ações em rede de forma que haja um prosseguimento do cuidado ao território onde as pessoas circulam e se relacionam. A proposta de cuidado de CAPSad, quando é voltada para o interior dos serviços e não para o território de vida, corre o risco de promover novas formas de institucionalização, além do afastamento da rede de apoio social na comunidade e a restrição de outras possibilidades de profissionalização, trabalho e lazer.

Portanto, é preciso repensar e reorganizar os serviços a partir do cotidiano e das relações estabelecidas dos usuários abusivos de álcool no território, considerando os movimentos e as redes que são conectadas em suas vidas. Esses movimentos extrapolam as paredes dos serviços e a atuação dos profissionais. Com isso, também precisam ser planejados em rede, principalmente de forma articulada com a atenção básica, assistência social e o setor judiciário.

Também fica em destaque neste estudo a parceria estabelecida entre o CAPSad e a Lokomotiva, configurando-se como estratégia que facilita a manutenção no tratamento e o processo de alta dos usuários do CAPSad para o território.

Quanto às outras redes sociais, destaca-se a escola como uma possibilidade de alcance dos objetivos de interação social; o ambiente domiciliar como um local de apoio, que proporciona a sensação de proteção, inclusive em momentos que sente vontade de beber; além desses, o desenvolvimento de atividades prazerosas que envolvem interação social na companhia da família ou amigos, o que demonstra que há outras atividades capaz de promover prazer e entretenimento. 
Com relação aos vínculos de amizade, o estudo aponta que os amigos são considerados companhias importantes, que promovem descontração, momentos de conversa, sentimentos de aceitação e valorização. Entretanto, a presença de outros usuários de drogas na rede social e na família aparece como um fator que pode levar à recaída.

$\mathrm{O}$ tratamento que objetiva a diminuição do uso de drogas requer a estruturação de recursos interacionais, portanto, vínculos deverão ser rompidos, como as amizades e companheiros de uso, outros deverão ser fortalecidos ou reatados, como os familiares, geralmente desgastados devido à dependência, e outros deverão ser construídos, como novas amizades e laços afetivos (Silva \& Ferigato, 2017, Mazaro et al., 2020).

Dessa forma, considera-se importante conhecer a rede social das pessoas que, devido ao uso prejudicial do álcool, estão assistidas na atenção psicossocial, pois mostra que não se reduzem à situação de saúde e o quão esses pontos na rede social podem apoiar no processo de recuperação. Facilitam a construção e ampliação de relacionamentos saudáveis que fortaleçam as estratégias de redução de danos, reinserção social e melhoria na qualidade de vida dos usuários de álcool.

\section{Conclusão}

A rede social das pessoas que fazem uso abusivo de álcool participantes deste estudo é composta pela família, amigos, CAPSad, espaço de geração de renda, trabalho, escola, ambiente domiciliar e atividades de lazer.

A família é descrita como um dos principais vínculos conflituosos, e também aparece nos vínculos fortes. Outros vínculos fortes incluem o CAPSad, a igreja, a Lokomotiva e as atividades de lazer. São aqueles com o qual o usuário se sente aceito, valorizado e apoiado afetivamente. Dessa forma, considera-se que as estratégias de cuidado devem considerar o tripé da reabilitação psicossocial- habitat, rede social e trabalho, uma vez que esses espaços aparecem como vínculos fortes, considerados potentes no apoio social dessas pessoas.

Evidencia-se que a rede social das pessoas que fazem uso abusivo de álcool não é restrita a um determinado serviço, pessoa, ou instituição especifica. É uma rede diversificada, que conta com recursos comunitários que auxiliam na manutenção da abstinência como a igreja/espiritualidade. Há também outros recursos que auxiliam na inserção do mercado de trabalho através da profissionalização e ainda com serviços de saúde que contribuem para a manutenção do tratamento.

O CAPSad é um importante recurso na rede social de pessoas que fazem uso abusivo de álcool, pelo apoio nos momentos difíceis do tratamento, além de suprir necessidades como alimentação, medicação, aceitação e afeto, e por propiciar novas sociabilidades. Portanto, considerando a relevância do CAPSad na rede social, é necessário que esse serviço assuma um papel estratégico de cuidado, incluindo o núcleo familiar nas propostas e acionando outros recursos da rede social e de saúde, de acordo com as necessidades e singularidades de cada caso.

Como limites deste estudo, destaca-se o fato de os sete participantes terem realizado o tratamento no CAPSad, o que impossibilitou conhecer outras realidades, entre elas a de pessoas com problemas relacionadas ao álcool que nunca fizeram tratamento em serviço especializado de saúde mental.

\section{Referências}

Alvarez, S. Q., Gomes, G. C., Oliveira, A. M. N. \& Xavier, D. M. (2012). Grupo de apoio/suporte como estratégia de cuidado: importância para familiares de usuários de drogas. Rev. gaúcha enferm., 33(2). http://dx.doi.org/10.1590/S1983-14472012000200015

Bard, N.; Antunes, B., Roos, C. M., Olschowsky, A. \& Pinho, L. B. (2016). Stigma and prejudice: the experience of crack users. Rev Latino-Am Enfermagem, 24(e2680). https://doi.org/10.1590/1518-8345.0852.2680.

Belotti, M., Fraga, H. L. \& Belotti, L. (2017). Família e atenção psicossocial: o cuidado à pessoa que faz uso abusivo de álcool e outras drogas. Cad. Bras. Ter. Ocup., 25(3). https://docs.bvsalud.org/biblioref/2018/02/879513/1727-8183-1-pb.pdf.

Beraldo, L., Gil, F., Ventriglio, A., Andrade, A. G., Silva, A. G., Torales, J., Gonçalves, P. D., Bhugra, D. \& Maia, J. M. C. (2019). Spirituality, Religiosity and Addiction Recovery: Current Perspectives. Curr Drug Res Rev., 11(1). http://dx.doi.org/10.2174/187447371166618061207595. 
Fagundes Junior, H. M., Desviat, M. \& Silva, P. R. F. (2016). Reforma Psiquiátrica no Rio de Janeiro: situação atual e perspectivas futuras. Ciênc saúde colet., 21(5). https://doi.org/10.1590/1413-81232015215.00872016.

Ferreira, T. P. S., Sampaio, J., Oliveira, I. L. \& Gomes, L. B. (2019). A família no cuidado em saúde mental: desafios para a produção de vidas. Saúde debate 43(121). http://dx.doi.org/10.1590/0103-1104201912112

Fitzpatrick, K. M. (2016). How Positive Is Their Future? Assessing the Role of Optimism and Social Support in Understanding Mental Health Symptomatology among Homeless Adults. [Quão positivo é o futuro deles? Avaliando o papel do otimismo e do apoio social na compreensão da sintomatologia da saúde mental entre adultos sem-teto]. Stress and health, 33(2). https://doi.org/10.1002/smi.2676.

Instituto Brasileiro de Geografia e Estatística (IBGE). (2017). Estimativas da população residente para os municípios e para as unidades da federação brasileiros com data de referência $1^{o}$ de julho de 2017. Rio de Janeiro: Instituto Brasileiro de Geografia e Estatística. https://www.ibge.gov.br/estatisticas/sociais/populacao/9103-estimativas-de-populacao.html?=\&t=resultados.

Kantorski, L. P., Cardano, M., Borges, L. R. \& Machado, R. A. (2020). Cotidiano como eixo de intervenção em saúde mental. Esc Anna Nery Rev Enferm, 25(1). https://doi.org/10.1590/2177-9465-EAN-2019-0373.

Marques, M. V., Silva Junior, D. N. S., Santos, E. G. O., Santos, S. S. A. N. S., Neves, S. M. B. \& Amador, A. E. (2020). Distribuição espacial das mortes atribuíveis ao uso de álcool no Brasil. Journal of Health \& Biological Sciences, 8(1), 1-11. http://dx.doi.org/10.12662/2317-3076jhbs.v8i1.2934.p1-11.2020

Martins, A. O; Ricci, E. C., Emerich, B. F. \& Campos R. O. (2018). Trabalho como estratégia de reabilitação social: desafios e potencialidades de uma oficina de trabalho. Rev Psicol UNESP, 17(2). https://www.fcm.unicamp.br/fcm/sites/default/files/2020/page/trabalho_como_estrategia_de_reabilitacao_social.pdf.

Mazaro, L. M., Matsukura, T. S. \& Lussi, I. A. O. (2020). Economia solidária como estratégia de inclusão social pelo trabalho no campo da saúde mental: panorama nacional. Cad Bras Ter Ocup., 28(1). https://doi.org/10.4322/2526-8910.ctoao1880.

Minayo, M. C. S. (2010). O desafio do conhecimento: pesquisa qualitativa em saúde. (12a ed.). Hucitec.

Ministério da Saúde (BR). (2015). Saúde Mental em Dados. Brasília: Ministério da Saúde https://www.mhinnovation.net/sites/default/files/downloads/innovation/reports/Report_12-edicao-do-Saude-Mental-em-Dados.pdf

Rodrigues, T. F. C. S., Sanches, R. C. N., Oliveira, M. L. F., PINHO, L. B. \& Radovanovic, C. A. T. (2018). Feelings of families regarding drug dependence: in the light of comprehensive sociology. [Sentimentos das famílias em relação à dependência de drogas: à luz da sociologia abrangente]. Rev bra enferm., 71(5). https://www.scielo.br/pdf/reben/v71s5/0034-7167-reben-71-s5-2272.pdf.

Romanini, M.; Guareschi, P. A \& Roso, A. (2017). O conceito de acolhimento em ato: reflexões a partir dos encontros com usuários e profissionais da rede. Saúde debate, 41(113). https://doi.org/10.1590/0103-1104201711311.

Saiz, M. J. S; Chacón, R. M. F; Abejar, M. G., Parra, M. D. S; Valentín, M. J. D \& Yubero, S. (2020). Perfil de consumo de drogas em adolescentes. Factores protectores. Soc Esp Med Rural Gen., 46(1). http://dx.doi.org/10.1016/j.semerg.2019.06.001.

Sanches, L. R. \& Vecchia, M. D. (2018). Reabilitação psicossocial e reinserção social de usuários de drogas: revisão da literatura. Psicol Soc., 30(e178335). https://doi.org/10.1590/1807-0310/2018v30178335.

Silva, A. P. D \& Ferigato, S. H. (2017). Mental health and work: dialogues on rights, desire and need for access. [Saúde mental e trabalho: diálogos sobre direitos, desejo e necessidade de acesso]. Cad Bras Ter Ocup., 25(4). https://doi.org/10.4322/2526-8910.ctoAO0951.

Silva, M. A. B. \& Abrahão, A. L. (2019). Política de atenção integral aos usuários de álcool e outras drogas: uma análise guiada por narrativas. Interface Comunicação, Saúde, Educação, 24(e190080). https://doi.org/10.1590/interface.190080.

Silva, L. L. P., Almeida, A. B. \& Amato, T. C. (2019). A perspectiva dos profissionais sobre o processo de alta de pacientes do Caps - AD: critérios e dificuldades. Saúde Debate, 43(122). https://doi.org/10.1590/0103-1104201912213.

Souza, J., Kantorski, L. P., Vasters, G. P. \& Luis, M. A. V. (2011). Rede social de usuários de álcool, sob tratamento, em um serviço de saúde mental. Rev Latino-Am Enferm; 19(1). https://doi.org/10.1590/S0104-11692011000100019.

Tan, Z.; Mun, E. Y., Nguyen, U. S. D. T. \& Walters, S. T. (2021). Increases in social support co-occur with decreases in depressive symptoms and substance use problems among adults in permanent supportive housing: an 18-month longitudinal study. [Aumentos no apoio social co-ocorrem com diminuições nos sintomas depressivos e problemas de uso de substâncias entre adultos em habitação de apoio permanente: um estudo longitudinal de 18 meses]. BMC Psychol, 9(6). https://doi.org/10.1186/s40359-020-00507-0.

World Health Organization (WHO). (2018). Global status report on alcohol and health. [Relatório da condição global de álcool e saúde]. https://www.who.int/substance_abuse/publications/global_alcohol_report/en/

Wright, L. M. \& Leahey, M. (2012). Enfermeiras e Famílias: um guia para avaliação e intervenção na família. (5a ed). Roca.

Zerbetto, S. R; Santile, N., Galera, S. A. F; Acorinte, A. C \& Giovannetti, G. (2017). Religiosity and spirituality: mechanisms of positive influence on the life and treatment of alcoholics. Esc Anna Nery Rev Enferm., 21(1). http://dx.doi.org/10.5935/1414-8145.20170005. 